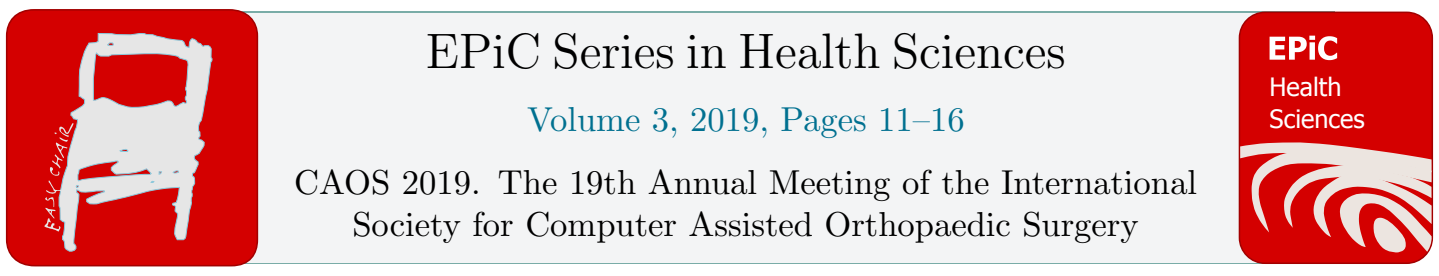

\title{
A Method for Validation and Error- Quantification of 3D-Printed Models for Acetabulum Fracture Surgery
}

Sebastian Andress ${ }^{1}$, Felix Achilles ${ }^{1}$, Eduardo M. Suero MD ${ }^{1}$, Christopher Becker MD ${ }^{1}$, Christoph Linhart MD ${ }^{1}$, Axel Greiner MD ${ }^{1}$, Bianka Rubenbauer $\mathrm{MD}^{1}$, Christian Kammerlander MD ${ }^{1}$, Wolfgang Böcker $\mathrm{MD}^{1}$ and Simon

Weidert $\mathrm{MD}^{1}$

${ }^{1}$ Klinikum der Universität München, Klinik für Allgemein-, Unfall- und

Wiederherstellungschirurgie, Munich, Germany

sebastian.andress@med.uni-muenchen.de,

simon.weidert@med.uni-muenchen.de

\begin{abstract}
Despite the increasing use of 3D printing in orthopedic surgery, there is no established method for the validation of a printed bone model. A printing error could potentially lead to complications, especially when the model is used for surgery planning. We aimed to validate a series of 33 acetabular fractures with a novel method by detailed analysis of model errors.

After applying a surface filter on both the patients model and the 3D printed model, a two-step registration consisting of landmark-based pre-registration and ICP-registration, was slightly varied and conducted 100 times, yielding a mean surface deviation and standard deviation for each model surface point.

We furthermore showed that more than 30 random ICP-registration points lead to a sufficiently good registration, and that the variation of the pre-registration is small enough to guarantee a stable ICP registration result.

Deviations caused by failed registration are unveiled by a large standard deviation of the 100 registration routines. For the fracture series, the mean surface deviation error between the printed model and the patient model was less than $1 \mathrm{~mm}$ (median $=0.7 \mathrm{~mm}$; $95 \% \mathrm{CI}=0.68-0.72 \mathrm{~mm}$ ). The largest errors were found in areas with remaining support structure material, on printbed-facing model sides and on abrasive surface regions. By visualizing the residual registration error, it was possible to clearly detect surface deviations and to quantify them.
\end{abstract}




\section{Introduction}

The accuracy of 3D models in orthopedic surgery is crucial if used for operative planning, especially when used as a template for pre-bending implants or it for intraoperative guiding purposes [1], [2]. Only few research has been done so far to validate the result of 3D printed fracture models.

As stated by George et al. the alignment is paramount for the comparison of the initial and printed model; thus, mostly manual and unstandardized method are described for this systematic error [3]. Artefacts in imaging data, faulty segmentation or flaws in the conversion to surface mesh data can have an adverse effect on the print accuracy. Moreover, the printing process itself or manual post-processing may lead to inaccuracies.

We hypothesize that for 3D printed bone fractures there are more factors that lead to printing errors. To be able to analyze shape deviations of the $3 \mathrm{D}$ prints in detail, we have established a method that allows for verification of the shape of a 3D printed model with complex acetabular fractures by comparing the models surface to the original model generated from computed tomography (CT) scans. For our analysis, we retrospectively applied the method on 33 unilateral acetabular fracture models from 32 patients.

\section{Methods}

All patients presented in a level 1 trauma center; preoperative CT scans with $0.5-1.25 \mathrm{~mm}$ slice thickness were generated (Siemens SOMATOM Force). We processed the datasets with a software solution previously developed by our group [4]. It creates an initial segmentation which is then refined with a shrink-wrap based surface filter that removes noisy internal structures, yielding a clean surface model. The resulting 3D mesh model was printed with a consumer-grade Fused Deposition Modeling printer (Ultimaker $2+$ ) with $0.21 \mathrm{~mm}$ layer height using polylactide (PLA) material.

For the subsequent analysis, the printed models were CT-scanned using a lung kernel for better airto-PLA contrast, reconstructed with $0.75 \mathrm{~mm}$ slice thickness and processed with the surface filter. As a result, we obtained a representation of both physical models as 3D surface meshes, which were then compared in the validation experiments.

Surface meshes are digital models which consist of 3D points, so called vertices, that are connected as triangles. We determine the surface distortion by computing the closest distance of each patient mesh vertex to the print mesh and averaging over the absolute distances.

Since distance deviations of the models can be explained both by shape defects and by ambiguous registration results, it is crucial to separately consider these factors.

By computing multiple randomly disturbed registrations and averaging the result, we try to eliminate registration as a source for model deviations. Our method consists of a two-step registration process:

1. Semi-automatic landmark pre-registration by selecting corresponding landmarks on both meshes. Attempting to mimic for inter-rater errors, the landmarks are slightly disturbed for every registration.

2. Then, an iterative closest points (ICP) algorithm is run to refine the registration. By using the ICP algorithm, we improve the registration based on surfaces rather than based on sparse landmarks. This is in accordance with the desired goal of the analysis, which is to evaluate surface deviations.

We used an implementation of the ICP algorithm that randomly selects a subset of vertices on one model and matches it to their nearest neighbors on the other model in each iteration. The optimal number of randomly selected points for the ICP algorithm was determined by repeated registrations, each time 
with the print model starting at a predefined shifted position $\left(10 \mathrm{~mm}\right.$ and $10^{\circ}$ in all directions), increasing the number of ICP points (Fig. 1A).

The influence of the landmark-based pre-registration and resulting error was measured in order to verify that our introduced random disturbance did not lead to wrong pre-registrations. A duplicate of the patients surface model was increasingly shifted (rotational and translational). Each time an ICP registration with the determined optimal number of vertices was computed and the remaining translational and rotational errors were calculated (Fig. 1B).

Finally, as the main comparison method, we performed the registration and calculated the accuracy of all printed models to unveil areas of surface deviations (Fig. 1C).

\section{Results and Discussion}

We found that $>30$ random vertices at ICP registration were sufficient to minimize deviation error between patient and print model (Fig. 1A). In the following experiments, 100 vertices were used. Furthermore, we quantified the significant effect of initial pre-registration on the final ICP registration (Fig. 1B) and found that initial rotational error had a greater influence on the final result compared to translational error.

The main validation of the models showed a wide range of both the mean distance as well as the standard deviation. Fig. 2 shows a "cold to hot" heatmap with some of our findings. Summarized, we were able to see the following influencing factors:

- Remaining support structures below the print lead to large errors, as also stated by RSNA [5]. Thus, model sides for implant fitting should be printed without support structures, i.e. on the upwards facing side of the print; other solutions are dissolving material or powder bedded print techniques [6]. Also, floor facing model sides often show a deviation due to inaccurate first printing layers, which bridge the gap between the support structures and the actual model. (Fig. 2, ID 25 \& 16)

- The maximum vertex error is not significant for the overall model deviation, since it is mainly is caused by small artefact particles that stick out from the model (Fig. 2, ID 21)

- An abrasive surface, for example caused by poor segmentation results, leads to a huge standard deviation of the surface distances, because no stable registration position can be found. (Fig. 2, ID 15)

While the maximum surface deviation, due to a small shape artifact, was at $9 \mathrm{~mm}$ distance, all our models showed a mean surface error of less than $1 \mathrm{~mm}$ (median $=0.7 \mathrm{~mm} ; 95 \% \mathrm{CI}=0.68-0.72 \mathrm{~mm}$ ).

\section{Conclusions}

Our method was able to demonstrate the types of deviations occurring in the printing process of bone fractures. Furthermore, it highlighted critical model areas, which should not be used for preoperative planning and implant fitting.

We have presented a novel method for precisely quantifying the error in 3D printed acetabulum fracture models. Specifically, after correct registration the mean surface distance can be used to verify the model shape as high artifact deviations only occur in very small regions.

This method aims to fill a current gap in the routine use of 3D printed models for trauma surgery by allowing the surgeon to precisely identify whether the printed model of a fracture is valid and whether it accurately represents the patient anatomy. 


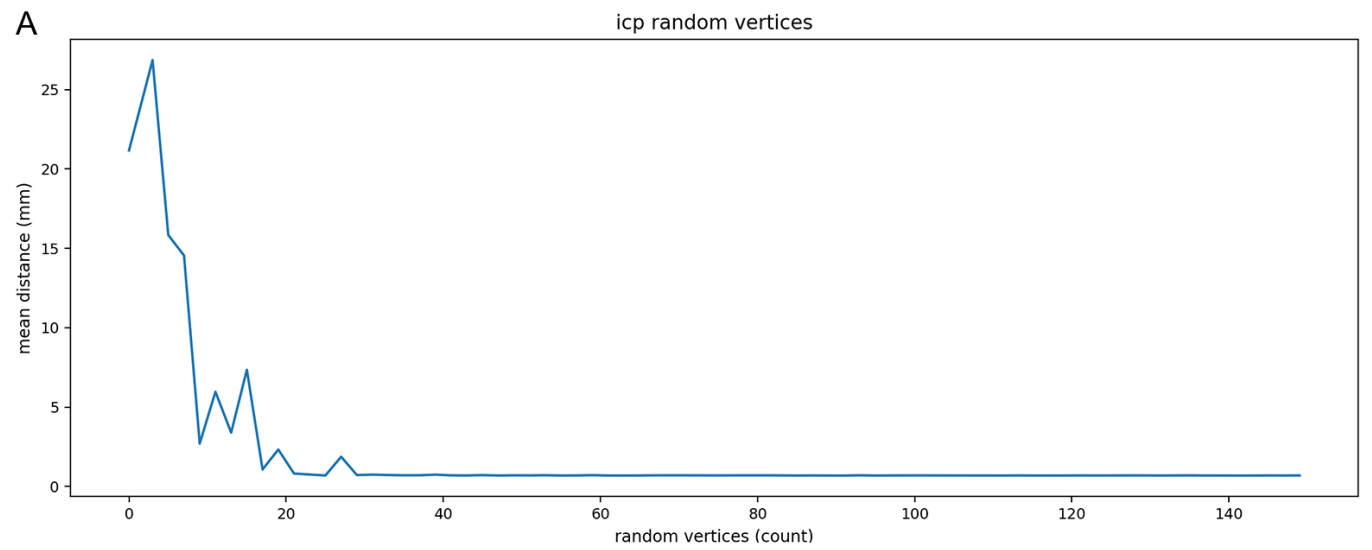

B
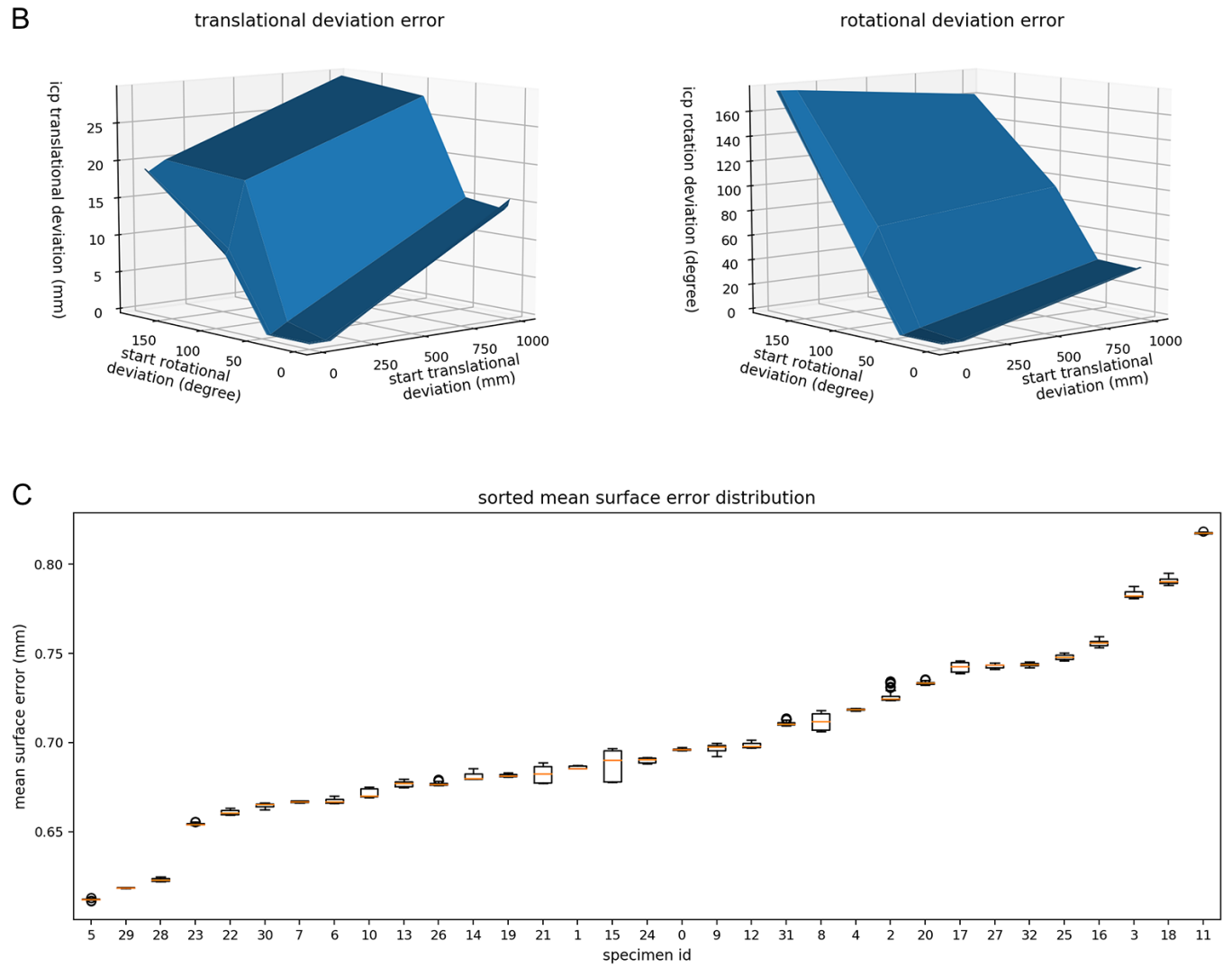

Figure 1: A: Experiment 1: Mean point deviation between patient and print model after ICP-registration depending on the configured number of random vertices. As early as 30 vertices already seem to lead to a good registration result. A pre-registration with an added artificial shift was done before the experiment $(10 \mathrm{~mm}$ translation, $10^{\circ}$ rotation) to ensure equal conditions. B: Experiment 2: Patient model was duplicated and artificially shifted (horizontal axes). Growing transformation distances and rotations (vertical axes) lead to larger translational deviation error (left) and rotation error (right). C: Validation Method: mean surface deviation and its standard deviation over the 100 registrations of the 33 Acetabular Fractures. 


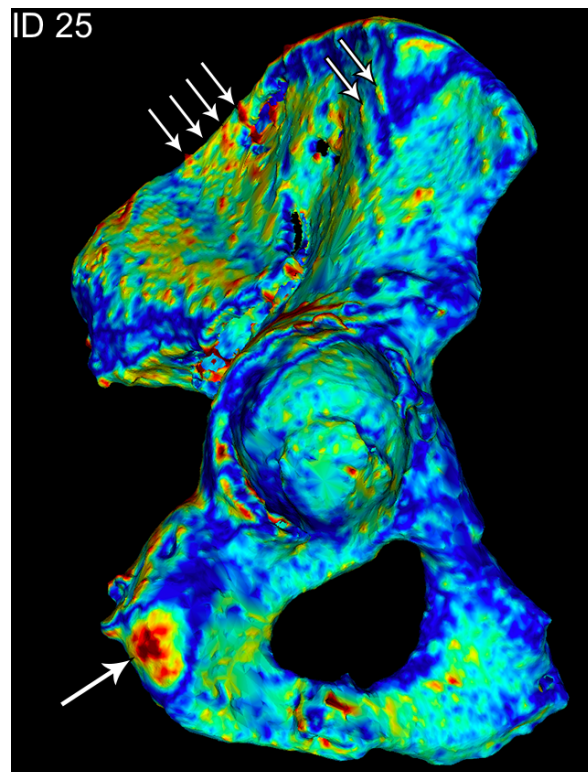

ID 16
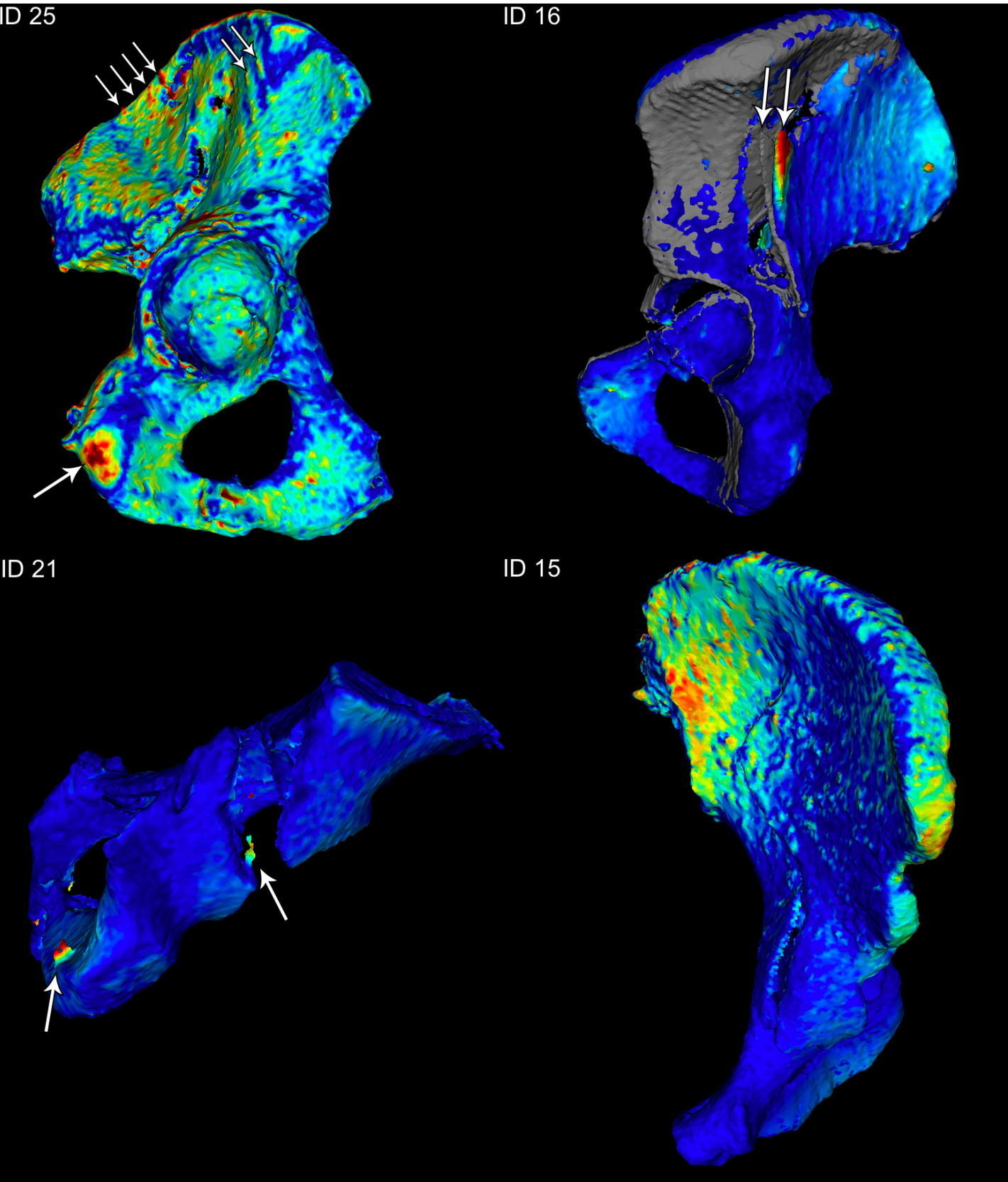

Figure 2: Color mapped surface distances and standard deviation (ID 15), cold-to-hot mapped (e.g. 0-2 mm, 0 is deep blue, $2 \mathrm{~mm}$ and above is deep red). ID 25: Errors due to floor facing artefacts (single arrow) and support structures, visible as stripes (e.g. small arrows) [0-2 mm]. ID 16: Right picture side faced the printbed. Gray model: patents surface mesh, shown at one registration example. Fracture piece of the print model shifted to the bottom. (arrows) [0-6 mm]. ID 21: old support structures and inaccurate segmentation leads to artefacts, visualized by our method. [0-6 mm]. ID 15: Example for a large standard deviation on rough surface. If these hot areas also show a large mean surface distance, this supposed displacement error might be false-positive. 


\section{References}

[1] G. A. Brown, K. Firoozbakhsh, T. A. DeCoster, J. R. Reyna, and M. Moneim, "Rapid prototyping: The future of trauma surgery?," J. Bone Jt. Surg. - Ser. A, vol. 85, no. SUPPL. 4, pp. 49-55, 2003.

[2] L. Maini, A. Sharma, S. Jha, A. Sharma, and A. Tiwari, "Three-dimensional printing and patient-specific pre-contoured plate: future of acetabulum fracture fixation?," Eur. J. Trauma Emerg. Surg., vol. 44, no. 2, 2018.

[3] E. George and P. Liacouras, "Measuring and Establishing the Accuracy and Reproducibility of 3D Printed Medical Models 1," no. 5, 2017.

[4] S. Andreß, U. Eck, C. Becker, A. Greiner, B. Rubenbauer, and S. Weidert, "Automatic Surface Model Reconstruction to Enhance Treatment of Acetabular Fracture Surgery with 3D Printing Sebastian Andreß, Ulrich Eck, Christopher Becker, Axel Greiner, Bianka Rubenbauer, Christoph Linhart, Simon Weidert," 2011.

[5] L. Chepelev et al., "Radiological Society of North America ( RSNA ) 3D printing Special Interest Group ( SIG ): guidelines for medical 3D printing and appropriateness for clinical scenarios," 2018.

[6] F. Rengier et al., "3D printing based on imaging data: review of medical applications," Int. $J$. Comput. Assist. Radiol. Surg., vol. 5, no. 4, pp. 335-341, Jul. 2010. 